
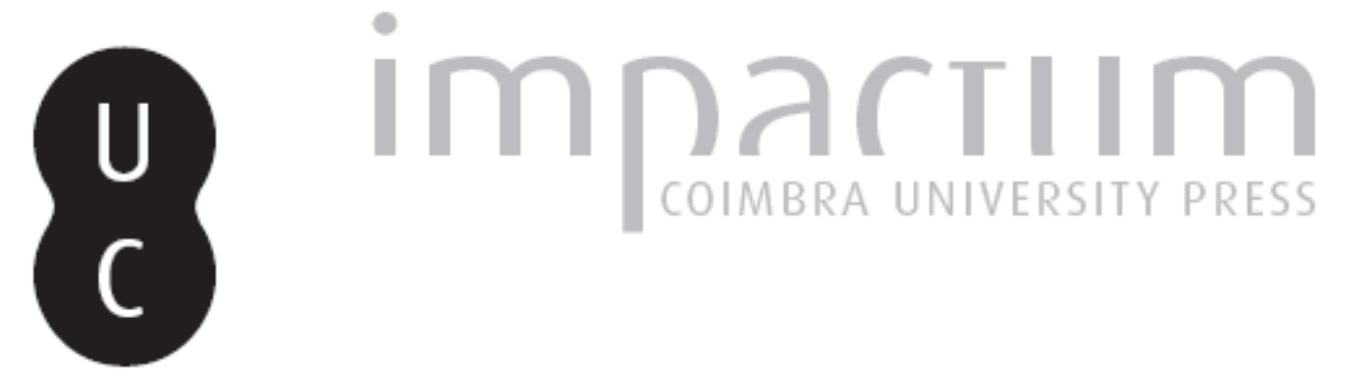

L'archeologia sperimentale e la preistoria: un contributo alla conoscenza del passato Autor(es): $\quad$ Giardino, Claudio

Publicado por: Faculdade de Letras da Universidade de Coimbra

URL persistente:

URI:http://hdl.handle.net/10316.2/36401

DOI:

DOI:http://dx.doi.org/10.14195/1647-8657_51_1

Accessed : $\quad$ 26-Apr-2023 13:56:43

A navegação consulta e descarregamento dos títulos inseridos nas Bibliotecas Digitais UC Digitalis, UC Pombalina e UC Impactum, pressupõem a aceitação plena e sem reservas dos Termos e Condições de Uso destas Bibliotecas Digitais, disponíveis em https://digitalis.uc.pt/pt-pt/termos.

Conforme exposto nos referidos Termos e Condições de Uso, o descarregamento de títulos de acesso restrito requer uma licença válida de autorização devendo o utilizador aceder ao(s) documento(s) a partir de um endereço de IP da instituição detentora da supramencionada licença.

Ao utilizador é apenas permitido o descarregamento para uso pessoal, pelo que o emprego do(s) título(s) descarregado(s) para outro fim, designadamente comercial, carece de autorização do respetivo autor ou editor da obra.

Na medida em que todas as obras da UC Digitalis se encontram protegidas pelo Código do Direito de Autor e Direitos Conexos e demais legislação aplicável, toda a cópia, parcial ou total, deste documento, nos casos em que é legalmente admitida, deverá conter ou fazer-se acompanhar por este aviso. 


\section{CONIMBRIGA}

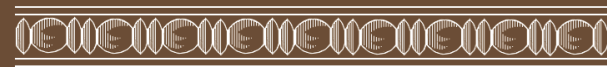

TIIDNIDN

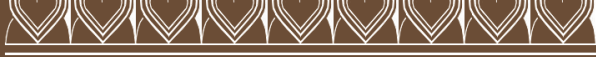

INSTITUTO DE ARQUEOLOGIA

VOLUME LI • 2012

FACULDADE DE LETRAS UNIVERSIDADE DE COIMBRA 


\author{
Claudio Giardino \\ Università del Salento - Lecce (Italia) \\ claudiogiardino@hotmail.it
}

\title{
L'ARCHEOLOGIA SPERIMENTALE E LA PREISTORIA: UN CONTRIBUTO ALLA CONOSCENZA DEL PASSATO
}

\author{
EXPERIMENTAL ARCHAEOLOGY AND PREHISTORY: \\ A CONTRIBUTION TO KNOWLEDGE OF THE PAST \\ “Conimbriga", LI (2012) 5-33
}

RIASSUNTO: La nascita dell'archeologia sperimentale coincide con quella degli studi scientifici di preistoria; ne sono stati pionieri studiosi scandinavi e inglesi, come S. Nilsson, J. Evans, J. Lubbock e A. Pitt Rivers. Essi applicarono il metodo sperimentale per studiare il funzionamento e le tecniche di produzione di manufatti preistorici, come gli strumenti in selce. In questo si servirono dei dati provenienti dalle indagini etnologiche: archeologia sperimentale ed etnoarcheologia sono state quindi sin dall'inizio strettamente associate.

Per le sperimentazioni del XIX secolo gli strumenti musicali protostorici - come carnyx celtici e lurer nordici - costituirono oggetto di particolare interesse.

L'esperimento archeologico deriva da scientifico, da cui però diverge per alcuni aspetti. Una delle principali diversità fra $i$ due è che in quello archeologico il risultato positivo non prova l'attendibilità di una teoria, che può essere dimostrata unicamente dalle evidenze archeologiche. Un obiettivo primario è la riproduzione delle catene operative, anche allo scopo di trarne dati di natura socio-culturale.

L'esame analitico dei residui prodotti nel corso dell'esperimento costituisce un elemento basilare per verificare la sua ripetibilità e compararlo con il record archeologico.

Conimbriga, 51 (2012) 5-33 
L'archeologia sperimentale è soggetta a rispecchiare le tendenze ideologiche e le mode culturali del momento; questo è particolarmente evidente nelle ricostruzioni di abitazioni o di villaggi preistorici che sono state più volte tentate sin dalla metà dell'Ottocento, divenendo spesso dei musei all'aperto e dei centri sperimentali. Attualmente la situazione economica impone a questi centri di coniugare la vocazione di ricerca con esigenze pedagogiche e turistiche.

Parole chiave: Archeologia sperimentale; Pre-protostoria; Metodologia; Musei all'aperto.

ABSTRACT: The beginning of experimental archeology coincides with the beginning of the scientific studies of prehistory. Pioneers were Scandinavian and English scholars, such as S. Nilsson, J. Evans, J. Lubbock and A. Pitt Rivers; they applied the experimental method in order to study the working procedures and production techniques of prehistoric artifacts, such as the flint tools. They also utilized the data coming from ethnological investigations for that researches: experimental archeology and ethno-archeology have been closely related since the beginning.

The $19^{\text {th }}$ century experimenters showed special interest for the proto-historic musical instruments, such as Celtic carnyx and Nordic lurer.

The archaeological experiment grows out of the scientific one, but it differs for some respects. One of the main differences between them is that in the archaeological experiment the positive result does not prove the reliability of a theory, which can be demonstrated only by archeological evidence. A prime objective is the reproduction of the operational chains, also in order to obtain socio-cultural data.

The analytical examination of the residues produced during the experiment is a basic element to verify its repeatability and to compare it with the archaeological record.

Experimental archeology reflects the ideological and cultural trends of the historical periods; this is mainly clear in the rebuilding of prehistoric houses or villages. These reconstructions have been frequently tried since half of the nineteenth century and they have become often open air museums and experimental centers. At present, the economic situation requires to these centers to 
combine the vocation to research with pedagogical and tourist needs.

KeY WORDs: Experimental Archaeology; Pre-protohistory; Methodology; Open Air Museums. 


\section{L'ARCHEOLOGIA SPERIMENTALE E LA PREISTORIA: UN CONTRIBUTO ALLA CONOSCENZA DEL PASSATO}

In ricordo di Angelo Bartoli

“... così si costuma e conviene nelle scienze le quali alle conclusioni naturali applicano le dimostrazioni matematiche, come si vede ne i perspettivi, negli astronomi, ne i mecanici, ne i musici ed altri, li quali con sensate esperienze confermano i principii loro, che sono i fondamenti di tutta la seguente struttura ..."

(Galileo Galilei, Discorsi e dimostrazioni matematiche intorno a due nuove scienze attenenti alla mecanica \& $i$ movimenti locali, 1638)

\section{I pionieri}

La nascita dell'archeologia sperimentale ha luogo nella metà dell'Ottocento e coincide sostanzialmente con quella degli studi scientifici di preistoria: non è un caso che abbia in gran parte padri comuni, soprattutto provenienti dal mondo anglosassone e scandinavo. Ne sono stati pionieri studiosi quali Sven Nilsson (1787-1883), John Evans (1823-1908), John Lubbock (1834-1913) e Augustus Pitt Rivers (18271900), che intuirono precocemente le grandi potenzialità di organizzare degli esperimenti al fine di meglio comprendere le evidenze archeologiche. Vennero agevolati in questo dalla loro formazione di base, spesso legata alle scienze naturali.

Il metodo sperimentale venne applicato in particolare per studiare il funzionamento e le tecniche di produzione di alcuni particolari oggetti, come gli strumenti in selce preistorici, che gli scavi stavano incominciando a riportare alla luce.

Probabilmente il primo esempio concreto di applicazione di metodiche sperimentali è da ricercarsi nello studio pubblicato nel 1838 dal naturalista e archeologo svedese Nilsson "Skandinaviska nordens urinvånare" nel quale, utilizzando anche paralleli etnologici, venne proposta una ricostruzione delle attività di caccia e pesca nella Scandi- 
navia dell'età della pietra. L'edizione inglese dell'opera, dal titolo "The primitive inhabitants of Scandinavia", fu tradotta alcuni anni dopo, nel 1868, da Lubbock, che ne curò anche l'introduzione avendone compreso e apprezzato il valore sia sul piano informativo che metodologico (Nilsson 1868).

Anche l'archeologo e geologo britannico Evans si interessò particolarmente alla litica; nel 1859 sostenne, davanti alla Royal Society di Londra, la grande antichità degli strumenti in selce che incominciavano a venire alla luce in molti paesi d'Europa in associazione con faune ormai estinte. Sei anni più tardi lo scienziato inglese Sir John Lubbock - in seguito Lord Avebury - pubblicò la prima edizione della sua opera "Pre-Historic Times" (Lubbock 1865), nella quale veniva definitivamente superata l'idea, mutuata dai testi biblici, che l'uomo fosse vecchio di soli seimila anni, ancora accettata da vari studiosi fino a pochi anni prima. Lubbock propose inoltre di modificare il sistema delle tre età - Pietra, Bronzo, Ferro - elaborato nel 1836 dal danese Christian Jurgensen Thompsen, in quattro periodi, articolando l'età della pietra in Paleolitico e Neolitico (Lubbock 1900, 2-3; Daniel 1968, 40-41, 43, 47-48, 53, 65).

In stretta connessione con i loro studi pionieristici, sia Nilsson, che Evans e Lubbock si interessarono alla riproduzione dei manufatti in selce, al fine di esaminare i metodi di fabbricazione e il modo di impiego. Per studiare le tecniche di scheggiatura e le modalità di distacco della litica preistorica, Lubbock si servì anche delle competenze dei fabbricanti di pietre focaie per fucili, un'attività che era ancora attiva nell'Inghilterra vittoriana nella sola Grimes' Graves, nel Norfolk, dove venivano sfruttati gli stessi giacimenti che erano stati utilizzati nel Neolitico (Lubbock 1900, 78, 81-82). In quegli stessi anni l'archeologo danese Niels Frederik Bernhard Sehested (1813-1882) utilizzava delle asce in pietra nella ricostruzione di una casa preistorica in legno nel parco di Broholm, nella Danimarca meridionale.

Particolarmente originale e decisamente anticipatore è il contributo fornito agli studi sperimentali dal generale Pitt Rivers, il cui nome alla nascita era Lane-Fox. Egli si dedicò soprattutto alle tecniche di scavo e di registrazione dei dati, conducendo indagini con metodo e rigore a Cranborne Chase, un'area dell'Inghilterra meridionale ricca di monumenti pre-protostorici. Per meglio comprendere i problemi legati alle alterazioni post-deposizionali dei fossati dell'età del ferro, ne svuotò alcuni e, dopo averli lasciati esposti per alcuni anni, ne riprese poi 
l'esplorazione, così da analizzarne la disgregazione delle pareti. Questo importante filone di ricerca sarebbe stato ripreso e sviluppato solo molti anni dopo, nella seconda metà del XX secolo. Sperimentò inoltre l'impiego degli antichi attrezzi agricoli riutilizzando per dissodare il terreno alcuni di quelli rinvenuti durante gli scavi (Guidi 1988, 24, 51).

\section{Le ricerche sulla litica}

I pionieri ottocenteschi degli studi sperimentali furono principalmente attratti dalla litica come oggetto privilegiato dello loro indagini. Il loro interesse era anche stimolato dal sempre crescente afflusso di manufatti in pietra spesso assai simili a quelli preistorici nelle collezioni museali, provenienti specialmente dall'Africa e dall'Oceania. Il colonialismo e le esplorazioni geografiche attivamente praticati dalle potenze europee dell'epoca fornivano una notevole quantità di nuove conoscenze etno-antropologiche sulle popolazioni degli altri continenti (Fig. 1).

Come è evidente già nell'opera di Nillson, archeologia sperimentale ed etnoarcheologia furono quindi sin dall'inizio strettamente associate specie nelle indagini sull'impiego e la produzione degli strumenti in pietra, reperti che soltanto fino a pochi anni prima erano spesso stati considerati alla stregua di "scherzi di natura".

Nonostante l'interesse iniziale e la precoce applicazione delle indagini sperimentali nel campo della litica, per molto tempo questo settore della ricerca non ha avuto significative innovazioni che andassero al di là di una sempre migliore capacità di riprodurre lo strumentario antico. Per sviluppare nuove tematiche di indagine bisognerà attendere gli anni successivi al secondo conflitto mondiale. Come sottolineato dal francese François Bordes (1919-1981), è stata la ricerca sperimentale ad aver reso possibile una reale conoscenza delle tecniche di lavorazione della pietra, e quindi poter distinguere un oggetto prodotto casualmente da uno intenzionale. Oltre allo stesso Bordes, Jacques Tixier (1925-) e l'americano Don E. Crabtree (1912-1980) hanno considerevolmente contribuito allo sviluppo della sperimentazione, definendo le diverse tecniche di percussione e di finitura superficiale dello strumentario litico, fondamentali per l' inquadramento delle industrie preistoriche, e diffondendo anche in questo settore l'applicazione di metodi statistici (Bordes 1968, 22-31).

Conimbriga, 51 (2012) 5-33 


\section{Le indagini sugli strumenti musicali}

Durante il XVIII e il XIX secolo vennero ripetutamente scoperti strumenti musicali attribuibili all'età del Bronzo e del Ferro negli ambienti umidi della Scandinavia e delle Isole Britanniche. Questi rinvenimenti posero gli strumenti e la musica protostorici all'attenzione sia degli studiosi di antichità che di un più vasto pubblico, affascinati dall'idea di riascoltare antichissime sonorità.

I corni celtici (carnyx) erano già noti al mondo antiquario dalla loro raffigurazione su coniazioni romane di età repubblicana, come ad esempio quelli effigiati sui denari emessi da D. Iunius Brutus Albinus nel 49-48 a.C. (cfr. Grueber 1910, tav. XLIX: 17; Sutherland 1974, 96, 163) (Fig. 2). Eseguirne un'esatta riproduzione per testarne le caratteristiche sonore appariva quindi una sfida particolarmente stimolante. Un incoraggiamento a sviluppare le ricerche archeo-musicologiche era fornito dalle stesse condizioni di rinvenimento di alcuni esemplari, che erano stati trovati non solo pressoché integri, ma anche eccezionalmente ben conservati, tanto da consentire di essere suonati. Nella sola Irlanda circa un quarto del centinaio di reperti era in tali condizioni.

L'interesse per queste ricerche indusse sin dal XVIII secolo ad analizzare la lega metallica con cui i corni erano fabbricati, al fine di consentire un'ottimale riproduzione delle qualità acustiche, naturalmente legate al tipo di metallo utilizzato. A questo fine ne venne addirittura sacrificato uno, il Lincolnshire carnyx, che era stato trovato, fratturato in due pezzi, in Inghilterra nel 1768 nel letto del fiume Witham, presso Tattershall Ferry (Lincolnshire): esso venne fuso per esaminarne la lega (Piggott 1959, 20, tav. VI).

Certamente i lurer prodotti durante la tarda età del Bronzo in Danimarca, ma presenti anche in Scandinavia, in Lettonia e nella Germania settentrionale, sono fra i più imponenti e notevoli strumenti musicali della protostoria europea (Fig. 3). Si tratta di manufatti in lega di rame di grandi dimensioni, con un corpo serpeggiante lungo da 1 a 2 metri circa e terminante in una larga piastra rotonda decorata da coppelle. Il loro uso doveva essere prevalentemente o esclusivamente rituale, come è anche suggerito dalle modalità del loro rinvenimento, generalmente a coppie abbinate simmetricamente.

I primi lurer intatti vennero scoperti casualmente alla fine del Settecento. Dal 1797, quando un contadino ne trovò sei esemplari mentre estraeva la torba nella palude di Brudevaelte a Sjælland, la maggiore 
isola della Danimarca, ne sono stati rinvenuti una sessantina, fra integri e frammentari (Coles 1973, 13-14, 164-167). La sperimentazione e gli studi condotti sui questi eccezionali manufatti hanno permesso di riconoscere che essi erano in grado di produrre da 7 a 9 note; esperti trombettieri potevano ricavarne sino a 16 (Broholm et al. 1949, 81; Coles 1973, 166).

\section{L'archeologia sperimentale come disciplina}

Sebbene l'archeologia sperimentale sia stata empiricamente utilizzata sin dal XIX secolo al fine di chiarire le problematiche del mondo antico e in particolare della pre-protostoria, la disciplina è stata definita da un punto di vista teorico solo nel 1973, con la pubblicazione dello studio di John Coles "Archaeology by Experiment".

La sperimentazione archeologica costituisce un potente mezzo di conoscenza per affrontare lo studio del mondo antico, finalizzato al chiarimento delle problematiche di interpretazione che emergono dalla ricerca.

Essa fonda la sua struttura nel metodo sperimentale ben noto nelle materie scientifiche, dove si è venuto ad affermare sin dal Seicento, a partire da Galileo (cfr. Galilei 1638, giornata terza). Secondo tali principi l'enunciazione delle leggi scientifiche avviene mediante conferma sperimentale delle ipotesi che sono state formulate sulla base dell'osservazione ripetuta di un dato fenomeno.

Il metodo è costituito sostanzialmente da un procedimento articolato in più fasi, nelle quali all'osservazione di un dato fenomeno segue la raccolta di dati intorno ad esso, l'individuazione delle variabili significative e l'elaborazione di un'ipotesi esplicativa con una previsione da verificare; conseguentemente vengono ideati e realizzati gli esperimenti, al fine di verificare o meno la previsione ipotizzata. Sulla base dei risultati ottenuti l'ipotesi originaria può essere accettata o rigettata; nel primo caso è possibile formalizzare un modello teorico.

L'esperimento archeologico ha molti punti in comune con quello scientifico da cui trae origine, pur divergendo da esso per alcuni aspetti non secondari (Tab. 1). Esso ha lo scopo di verificare quelle ipotesi che possano fornire o aumentare le analogie con eventi antichi, o eventualmente di stimolare la formulazione di nuove (cfr. Mathieu 2002, 12). Anche l'esperimento archeologico, per essere considerato valido, deve 
sottostare ad alcune precondizioni, quali l'essere ripetibile, soddisfare i canoni disciplinari di riferimento ed essere progettato in modo da fornire risultati statisticamente accettabili e confrontabili con i record archeologici di partenza (Reynolds 1999; Comis 2004, 229-230).

Un elemento rilevante e peculiare della sperimentazione archeologica è che il materiale impiegato nella ricostruzione del processo debba essere analogo a quello disponibile localmente da parte delle antiche comunità sulle quali si sta conducendo l'indagine.

Talvolta per poter correttamente eseguire un esperimento valutandone e registrandone i parametri significativi (Fig. 4) può essere necessario eseguire preliminarmente una serie di prove in condizioni meno controllabili, ma indispensabili affinché si acquisisca la necessaria capacità operativa che permetta poi di portare a termine la replica del fenomeno indagato. Può mancare, a chi provi a riprodurre tecniche sconosciute perché ormai dimenticate da secoli, quella manualità e quelle nozioni di base - anche di dettagli apparentemente secondari - che erano invece parte integrante dell'esperienza e del bagaglio cognitivo dell'antico operatore. Tali tradizioni tecniche, tra l'altro, costituivano spesso un aspetto determinante e distintivo fra le diverse culture.

La valutazione dell'attività sperimentale non è tanto fornita dalla corretta riproduzione dei manufatti antichi, quanto piuttosto dall'esame analitico dei residui prodotti nel corso dell'esperimento. Solo il confronto fra le indagini archeometriche condotte su tali residui e quelle ottenute esaminando i materiali archeologici fornirà infatti una misura del successo o meno dell'operazione. Una corretta sperimentazione va quindi affiancata a metodologie analitiche spesso sofisticate, che consentano di parametrare l'esperimento sia nel corso del suo sviluppo, che nei suoi esiti finali, rapportandone i risultati a valori quantitativi, affinché sia così sarà possibile verificare appieno la sua ripetibilità $\mathrm{e}$ compararlo propriamente con il record archeologico di partenza.

\section{Archeologia sperimentale e catena operativa}

Uno dei più promettenti campi di indagine della moderna sperimentazione archeologica è lo studio dei rapporti di produzione e lavorazione, settore nel quale è indispensabile un approccio sistematico e multidisciplinare, tenendo conto non solo degli aspetti quantitativi, ma anche di quelli qualitativi del record archeologico. Obiettivo primario 
è la riproduzione delle catene operative, anche allo scopo di trarne dati di natura socio-culturale.

Il concetto di catena tecnico-operativa formulato da André Leroi-Gourhan (1964) costituisce uno dei più innovativi e originali strumenti teoretici per gli studi archeologici prodotti nella seconda metà del secolo scorso.

La catena operativa (chaîne opératoire) è la sequenza dinamica di azioni materiali fra loro concatenate che portano alla trasformazione fisica delle risorse naturali in manufatti, cioè in prodotti culturali (cfr. Dobres 2000, 154).

Si può analizzarla scomponendola nelle singole parti della sequenza: ad esempio nel caso della fabbricazione di un oggetto metallico in lega di rame, la catena include l'insieme delle fasi operative, dalla raccolta delle materie prime (sia il minerale cuprifero, che il combustibile e l'argilla per costruire il forno), ai diversi momenti della fabbricazione (estrazione del metallo dal minerale, alligazione, fusione in matrice, finitura), all'utilizzo dell'oggetto, alla sua eventuale riparazione (affilatura, restauro) e, eventualmente, alla sua rifusione, fino all'abbandono con il suo scarto definitivo. Ciascuna fase presenta una specifica valenza culturale: poiché gli individui in un gruppo dispongono di un certo numero di materie prime e di tecniche utilizzabili, l'identificazione delle scelte comunemente operate - cioè della sequenza operativa - permette di evidenziare le tradizioni tecniche del gruppo sociale e quindi di definire i diversi stili e le differenti strategie culturali messe in opera dalle diverse comunità per ottenere gli esiti desiderati.

L'archeologia sperimentale, provando a ricomporre concretamente le catene operative a partire dai dati archeologici, permette così di indagare sui comportamenti culturali delle società antiche.

Per la ricostruzione delle catene operative la sperimentazione deve necessariamente avvalersi degli apporti di altre discipline, come l'etnoarcheologia e l'archeometria. Il loro mutuo contributo alla corretta interpretazione delle sequenze costituisce oggi un indispensabile supporto delle tradizionali metodiche archeologiche, contributo che consente non solo di identificare gli indicatori archeologici, ma anche di interpretare correttamente i processi di formazione dei depositi antichi (Bellintani et al. 2003, 331).

Conimbriga, 51 (2012) 5-33 


\section{Realtà e possibilità potenziale: il caso delle navi Viking e Ra.}

Nel campo dell'archeologia sperimentale, a differenza che nell'esperimento scientifico classico, il risultato positivo non costituisce necessariamente una prova assoluta che il problema esaminato abbia quella specifica soluzione. È questa una delle principali diversità fra l'applicazione del metodo nel mondo delle discipline fisiche e in quello dei saperi storico-antichistici.

L'universo della cultura umana è infatti assai meno deterministico di quello delle scienze naturali: il successo della prova sperimentale indica quindi unicamente che quella spiegazione è possibile, non che sia quella reale e, soprattutto, non l'unica.

Un buon esempio è fornito dagli esperimenti sulla navigazione condotti dall'esploratore norvegese Thor Heyerdahl fra la fine degli anni Sessanta e i primi anni Settanta del Novecento allo scopo di verificare alcune teorie sulla migrazione dei popoli antichi (Heyerdahl 1971).

Già nell'Ottocento la sperimentazione nel campo della marineria aveva portato a ricreare antiche imbarcazioni che potessero tenere il mare. Allo scopo di dimostrare che gli antichi vascelli medievali vichinghi erano in grado di attraversare l'Atlantico, nel 1893 fu prodotta in Norvegia la Viking; essa venne fabbricata a Sandefjord, nel sud del paese, sulla costa dello Skagerrak, come fedele replica della Nave di Gokstad, un'imbarcazione vichinga lunga $24 \mathrm{~m}$ e databile al IX secolo che era stata rinvenuta nel 1880 in una tomba a tumulo nella contea norvegese di Vestfold (Fig. 5). Il problema di trovare tronchi di quercia delle dimensioni necessarie per la chiglia e l'albero, ormai irreperibili in Scandinavia, venne risolto importandoli dal Canada. La Viking, al comando del capitano Magnus Andersen, veleggiò da Oslo a Bergen, da cui ad aprile iniziò il suo viaggio nell' Atlantico settentrionale, raggiungendo l'isola canadese di Terranova in soli 28 giorni. Costeggiando la Nuova Scozia e il Massachusetts arrivò poi a New York, per pervenire infine a Chicago, dove venne esibita alla World's Fair Colombian Exposition (Andersen 1895; Ellmers 2005, 49; http://home.online.no/ joeolavl/viking/viking-kopi-gokstadskipet.htm\#Vikingef\%C3\%A6rden), esposizione realizzata per commemorare i 400 anni della scoperta di Colombo. In tal modo la Viking dimostrò come fosse possibile che $\mathrm{i}$ Vichinghi avessero raggiunto il continente americano già 500 anni prima, come narravano le saghe islandesi, che riferivano di varie spedizioni condotte intorno all'anno 1000 verso una terra ospitale chiamata 
Vinland. Tuttavia per oltre mezzo secolo l'ipotesi vichinga restò solo un'affascinante teoria, sebbene l'impresa della Viking ne avesse dimostrato la possibilità potenziale: solo negli anni ' 60 del XX secolo, gli scavi archeologici portarono alla luce un insediamento vichingo dell' $\mathrm{XI}$ secolo a L'Anse aux Meadows nel nord di Terranova, fornendo così la prova definitiva della correttezza di questa teoria (Ingstad 1977; Ingstad 1985; Nydal 1989, 976, 984).

Heyerdahl, allo scopo di dimostrare che gli Egizi sarebbero stati in grado di raggiungere con le loro navi le Americhe, costruì un'imbarcazione con canne di papiro sulla base di antiche raffigurazioni tombali e vascolari egiziane che mostravano navi a doppia punta, non dissimili da quelle ancora prodotte in alcune parti dell'Africa e dell'America meridionale. Per costruire la nave $R a I$ vennero utilizzate le tecniche di assemblaggio dello scafo praticate sulle rive del lago Ciad, combinando così assieme dati mutuati da ricerche archeologiche ed etno-archeologiche. La nave era lunga 14 metri e partì dalle coste atlantiche del Marocco per raggiungere il Nuovo Continente. Dopo una cinquantina di giorni, però, le avverse condizioni del mare fecero naufragare la $R a$ a $970 \mathrm{~km}$ dall' isola di Barbados nelle Antille, dopo aver coperto oltre $4300 \mathrm{~km}$. Venne quindi realizzata una seconda nave, la $\mathrm{Ra} I I$, questa volta da fabbricata da indigeni del lago Titicaca, abili costruttori di barche di canne, che salpò anch'essa dalle coste marocchine. La nuova imbarcazione, lunga 12 metri, si dimostrò più solida della precedente $\mathrm{e}$ riuscì a raggiungere Barbados in 57 giorni.

L'esperimento, che incontrò un vasto interesse mediatico, dimostrò come una nave di papiro potesse resistere a un viaggio oceanico e che i venti e le correnti avrebbero potuto fornire la propulsione necessaria ad attraversare l'Atlantico, ma certamente non che il viaggio fosse stato effettivamente realizzato dagli Egizi nell'antichità. Il semplice fatto che un'azione sia potenzialmente possibile non implica infatti necessariamente che sia realmente avvenuta.

\section{Mode culturali e archeologia sperimentale: le strutture abitative pre-protostoriche.}

Come tutte le discipline umane, l'archeologia sperimentale è soggetta a rispecchiare le tendenze ideologiche e le mode culturali del momento. Questa caratteristica appare particolarmente evidente nel 
caso della ricostruzione delle strutture abitative che, per la loro complessità, richiedono la soluzione di molteplici problemi di ordine sia operativo, che estetico. La creazione di parchi archeologici e di musei all'aperto dove poter apprezzare e vivere strutture abitative pre-protostoriche in grandezza naturale, spesso realizzate utilizzando tecniche antiche, è stata una delle più precoci esperienze della sperimentazione archeologica e costituisce ancora oggi una delle principali attività del settore.

Purtroppo, però, ben poco si conosce delle abitazioni pre-protostoriche se non la pianta, che di norma costituisce la principale evidenza strutturale restituita dagli scavi, mentre l'alzato è andato quasi sempre perduto. Nella realizzazione di una replica è invece determinante la creazione delle parti superiori della casa, e in particolare delle pareti e del tetto.

Le urne a capanna del Bronzo Finale e della prima età del Ferro del Lazio e dell'Etruria sono dei veri e propri modellini di abitazioni protostoriche. Esse appaiono spesso decorate con motivi geometrici, dimostrando come il paramento murario dovesse talora in origine essere rifinito esternamente mediante pittura o graffiti, contribuendo in modo determinante all'aspetto visivo della casa. Tenendo conto anche di questi dettagli, niente affatto secondari, è stata realizzata la ricostruzione in scala reale della capanna di Fidene, a Roma, sulla base degli scavi condotti dalla Soprintendenza archeologica di Roma in quell'area negli anni fra il 1986 e il 1993 (De Santis et al. 1998). Il tetto doveva essere realizzato con tecniche e materiali - quali canne e frasche - tali da trattenere il calore, ma anche da permettere l'esalazione dei fumi che si producevano all'interno, magari con l'ausilio di apposite aperture, finalizzate pure all'illuminazione. Le capanne inoltre non dovevano essere prive di decorazioni, come si osserva ancora oggi ad esempio in Italia nella copertura dei trulli tradizionali della Puglia (Bietti Sestieri, De Santis 2004, 171, fig. 11).

Purtroppo le condizioni in cui si è conservato l'alzato sono del tutto eccezionali, come nel caso di Croce del Papa a Nola (Napoli), dove l'eruzione del Somma-Vesuvio ha consentito la sopravvivenza di strutture del Bronzo Antico ben conservate. Il villaggio, già in parte coperto da pomici e ceneri, fu investito subito dopo da un'alluvione fangosa che inglobò le capanne, realizzando un perfetto calco delle strutture lignee e in paglia, sia all'esterno che internamente (Vecchio et al. 2002, 21-25).

Conimbriga, 51 (2012) 5-33 
A causa dell'estrema scarsità di dati sulle parti in elevato, nella ricostruzione sperimentale delle case, vengono generalmente utilizzati solo metodi di costruzione e di carpenteria assai primitivi, sebbene lo studio dello strumentario del Bronzo Finale e dell'età del Ferro nonché i rari elementi in legno restituiti da ambienti umidi dimostrino come lo sviluppo delle tecniche di carpenteria non differisse in maniera sostanziale nella tarda protostoria da quello dei periodi storici (Audouze, Buchsenschutz 1991, 48; Bradford 1992, 320-322, 324-327; Thomson 1993, 397-398; Briggs 1993, 452). Molto verosimilmente una struttura abitativa di un oppidum celtico non doveva poi essere strutturalmente dissimile da una casa a graticcio dell'Europa centrale del XVIII o XIX secolo (Schmidt 1994, 17).

Poiché il record archeologico fornisce di norma unicamente dati planimetrici, la mancanza di informazioni ha spinto i realizzatori dei modelli sperimentali a creare strutture per lo più assai semplici e di sconcertante omogeneità: è infatti spesso arduo distinguere la ricostruzione di una capanna neolitica da una dell'età del Bronzo o finanche altomedievale. E invece esperienza comune poter abbastanza agevolmente distinguere lo stile di un vero edificio, ad esempio un'abitazione rinascimentale da una ottocentesca o una moderna casa di campagna tedesca da una consimile greca.

Questa stessa carenza ha inoltre portato a basare le repliche su quella che era la percezione ideale della preistoria nel momento in cui la replica stessa veniva eretta: la "ricostruzione" di un'abitazione o di un intero villaggio preistorico finiva così per riflettere soprattutto lo spirito del tempo (zeitgeist), rispecchiando in realtà come la cultura dominante in un determinato periodo storico leggesse - o desiderava venisse letta - la preistoria. La visione del passato infatti contribuisce in misura determinante a creare un senso di identità condivisa ed è stata a tal fine utilizzata - talora senza molti scrupoli - per giustificare e promuovere sistemi politici e sociali.

Le prime ricostruzioni a dimensioni reali di strutture preistoriche vennero prodotte nella seconda metà dell'Ottocento, seguendo una tradizione ancora pienamente legata al Romanticismo. Un esempio è offerto dalle palafitte realizzate in Svizzera nel Bally-Park di Schönenwerd ad Aarau, nel Canton Soletta, lungo il fiume Aare, il cui inizio data al 1868; il complesso si è poi pienamente sviluppato fra il $1890 \mathrm{e}$ il 1920. Nelle ricostruzioni allora proposte si manifestava con eviden$\mathrm{za}-\mathrm{e}$ in maniera acronica - l'immagine, cara all'ambiente germanico 
dell'epoca, di un mondo ideale teutonico, puro e sano, in contrapposizione a quello mediterraneo, decadente. La realizzazione è sostanzialmente improntata al mito del "buon selvaggio". La stessa concezione di vigore misto a purezza improntava del resto anche i dipinti a soggetto pre-protostorico, come le tele ad olio di Albert Anker "La palafitticola", del 1873, e quella di Otto Emanuel Bay del 1891 "La palafitta di Moosseedorfsee" (Bandi 1983, 22-23, Fig. 6-7). Un analogo spirito è alla base della creazione in Austria di quattro palafitte nel lago di Attersee da parte dell'associazione "Patria Tedesca" di Linz intorno al 1910. Il fine primario non era tanto un'esatta ricostruzione scientifica, quanto piuttosto stimolare l'interesse popolare nella preistoria a fini sostanzialmente nazionalistici (Schöbel 2004 a, 153, Fig. 3).

Ciò non toglie che alcune realizzazioni di quel periodo, come la fabbricazione di una casa neolitica a Rössen in Alsazia, costituissero anche significativi esperimenti archeologici, considerando che sino a quel momento la ricostruzione scientifica di strutture era stata quasi esclusivamente eseguita sulla carta o facendo ricorso a modellini in scala (Hahne 1919) (Fig. 6). L'impiego di analogie etnografiche, specie nella scelta delle tecniche costruttive, appare assai frequente in quegli anni ed è in stretto rapporto con il fenomeno dell'espansione coloniale europea che caratterizza gran parte del XIX secolo.

L'avvento delle dittature successivo alla prima guerra mondiale cambiò nettamente la prospettiva, come si rivela con particolare evidenza in Germania. Il Nazionalsocialismo voleva soprattutto documentare e diffondere l'eterna superiorità della razza ariana, sia dal punto di vista culturale che tecnologico, proponendo e divulgando un mitico passato glorioso. Il personaggio del "selvaggio felice" caro alle rivisitazioni ottocentesche fu così sostituito dall'attivo e vigoroso contadino germanico, mentre i paralleli costruttivi non vennero più tratti dall'etnografia extraeuropea, ma dal folklore tedesco. Finalità didattiche e di propaganda spinsero a realizzare parchi archeologici e musei all'aperto nel territorio del Reich.

Uno dei principali fu il Museo delle Palafitte di Unteruhldingen, nel comune di Uhldingen-Mühlhofen, sulla riva nord-occidentale del Lago di Costanza. Esso fu realizzato fra il 1922 e il 1941 e, con le sue ricostruzioni ideali di strutture preistoriche palafitticole in grandezza naturale, divenne un modello da imitare per sottolineare il profondo legame storico fra la popolazione e il patrimonio culturale del territorio (Ströbel 1939, 42). Proprio ad Unteruhldingen venne realizzato fra il 
1926 e il 1927 - quindi pochi anni prima dell'ascesa al potere di Hitler, nel 1933 - "Natur und Liebe" il primo film, muto, sulla vita nelle palafitte. Il parco museo è tuttora attivo e oggi offre ai visitatori la visita di una ventina di capanne del Neolitico e dell'Età del Bronzo (Schöbel 2004, 154, fig. 6; http://www.pfahlbauten.de).

\section{Archeologia sperimentale e musei all'aperto: tendenze e sviluppi attuali}

Nel secondo dopoguerra, e particolarmente a partire dagli anni Sessanta, i progetti di ricostruzione di abitazioni preistoriche assunsero un aspetto più propriamente legato all'archeologia sperimentale, venendo spesso realizzati all'interno di aree laboratorio particolarmente dedicate alla sperimentazione, come la Butser Ancient Farm presso Petersfield nell'Hampshire (Fig. 7), in Gran Bretagna e l'Historisk-Arkaeologisk Forsogscenter a Lejre, presso Roskilde in Danimarca.

Anche nei paesi dell'Europa orientale, i cui regimi erano improntati all'ideologia comunista, furono creati musei all'aperto, generalmente con il concorso di archeologi e di accademici. Le strutture antiche furono riprodotte con cura scientifica, sovente negli immediati pressi di abitati pre-protostorici e in stretta connessione con gli scavi, come a Biskupin in Polonia e a Brežno u Loun (Postoloprty) nell'attuale Repubblica Ceca (Fig. 8). In quest'ultimo sito, usato a fini didattici dall'Istituto di Archeologia dell'Università di Praga, accanto ad ogni ricostruzione era collocata la planimetria della struttura così come era emersa dall'indagine archeologica.

Talora sono state create delle strutture abitative preistoriche con il fine specifico di esaminare i fenomeni legati alla loro distruzione, come è avvenuto in un celebre esperimento condotto negli anni ' 60 a Roskilde, in Danimarca. La casa, una grande capanna dell'età del Ferro fabbricata sul modello di un'abitazione scavata negli anni Trenta, fu realizzata con grande cura, disponendovi dentro ceramiche e cesti per simulare la situazione reale. Essendo destinata a bruciare, al suo interno furono inserite delle termocoppie per registrare l'andamento delle temperature che si sarebbero raggiunte durante la combustione e ogni suo palo venne marcato con piastrine metalliche. Incendiata mediante delle fascine poste sul fianco della struttura, il fuoco riuscì a distruggerla completamente in 35 minuti; dopo sei mesi il sito fu scavato, fornendo 
dati di eccezionale interesse per le interpretazioni archeologiche (Nielsen 1966; Coles 1973, 64-66).

Fra i successivi esperimenti compiuti sulla distruzione di abitazioni va ricordato quello condotto nel 1989 da Pierre Pétrequin al Lago di Chalain, nel Giura: due palafitte neolitiche, ricostruite sulla base degli scavi condotti negli insediamenti di Chalain e di Clairvaux, corroborati da dati etno-archeologici e di archeologia sperimentale, vennero lasciate decadere naturalmente dopo alcuni anni per esaminare i fenomeni fisici legati al loro degrado (Pétrequin 1997, 100; Schöbel 2004 b, 231; Leuzinger 2004, 241).

Numerosi sono stati i centri nati nella seconda metà del secolo scorso in gran parte dei paesi dell'Unione Europea, spesso con contributi comunitari. Si possono, a titolo esemplificativo, ricordare l'Archéosite d'Auberchies in Belgio, il Parc Archeologique de Beynac e l'Archéodrome du Bourgogne a Beaune entrambi in Francia, Asparn an der Zaya in Austria, il Centro di Archeologia Sperimentale di Blera presso Viterbo e il Parco Archeologico della Terramara di Montale presso Modena in Italia.

Per sopravvivere, tutti questi centri hanno dovuto coniugare sin dall'inizio la vocazione di ricerca con esigenze pedagogiche e turistiche.

Tramontato il periodo dei sussidi pubblici alle attività culturali, queste debbono infatti necessariamente trovare sostentamento nel privato. I finanziamenti alle attività scientifiche vengono quindi dai servizi informativi offerti sia alle scuole di vario ordine e grado con seminari, laboratori, stage, che anche ad un più vasto pubblico che paga per assistere alle attività dei centri (cfr. Chevillot 1994, 38-39).

A causa della duplice natura imposta dal mercato, è un problema attuale quello di distinguere i centri a vera vocazione archeologico-sperimentale da quelle strutture volte pressoché esclusivamente a intercettare l'interesse dei visitatori, che tendono così a trasformarsi in una sorta di parchi tematici alla Disneyland.

Non è certo incoraggiante il triste esempio dell'Archéodrome di Beaune in Bourgogna, uno dei più prestigiosi d'Europa, costretto a chiudere dopo 25 anni di attività per motivi economic, dopo essere passato in gestione ad una società autostradale, la Société des Autoroutes Paris-Rhin-Rhône. Era stato, nel 1978, il primo parco francese ad aver riprodotto al suo interno case e monumenti dal Neolitico al periodo gallo-romano e a possedere un rilevante e ricco museo didattico desti- 
nato al largo pubblico; aveva inoltre ospitato convegni scientifici internazionali, contribuendo a promuovere la diffusione dell' archeologia sperimentale, anche con una apposita rivista, $C u+$ (Frère-Sautot 2011) (Fig. 9-10).

Fin dagli ultimi anni del secolo passato alcune associazioni, come la svizzera AEAS (Arbeitsgemeinschaft für Experimentelle Archäologie der Schweiz) hanno provato a enucleare gli elementi caratterizzanti di un vero parco sperimentale, mettendo in evidenza le cinque attività principali che esso dovrebbe comunque offrire (Leuzinger 2004, 238): 1) sperimentare situazioni basate sull'evidenza archeologica; 2) studiare e insegnare tecniche e abilità del passato; 3) offrire dimostrazioni o usare ricostruzioni a fini di ricerca, didattici o in mostre; 4) sviluppare esperimenti scientifici; 5) documentare, registrare e pubblicare i risultati delle esperienze eseguite.

Allo scopo di creare un accordo internazionale sulle principali linee guida di una comunità scientifica di archeologia sperimentale, nel 2001 si è costituita l'EXARC (European Exchange on Archaeological Research and Communication), a cui aderiscono attualmente un centinaio di strutture di tutto il continente (www.exarc.net). Con il suo statuto, essa mira a garantire un'elevata qualità sia nella didattica che nella ricerca scientifica, eticamente basata sulla correttezza sperimentale e sull'alto livello di documentazione (EXARC 2002, 7-8). All'EXARC appartiene, tra gli altri, in Italia, il Centro di Archeologia Sperimentale di Blera. Esso fonda i suoi introiti sulla didattica e sull'informazione archeologica, offrendo lavoro a vari archeologi, ma presta gratuitamente le sue strutture e le sue competenze a quegli studiosi che desiderino realizzare delle prove sperimentali, promuovendo inoltre convegni e incontri internazionali ai ricercatori del settore (Fig. 11). Questo centro italiano può oggi costituire un valido modello, come altre strutture consimili sparse in Europa, sia sul piano scientifico che occupazionale, in un momento in cui l'assenza di una reale presenza pubblica nel mondo della cultura archeologica disincentiva di fatto le attività di ricerca e lascia senza occupazione molti giovani specialisti capaci. 


\section{BIBLIOGRAFIA}

ANDERSEN M. 1895, Vikingefcerden: en illustreret beskrivelse af "Vikings" reise i 1893, Kristiania (Oslo).

Audouze F., Buchsenschutz O. 1991, Towns, Villages and Countryside of Celtic Europe, London.

Bandi H.-G. 1983, Immagini e riproduzioni di palafitte nel XIX secolo, in Palafitte: mito e realtà, Verona, pp. 15-24.

Bellintani P. (a cura di), Cesaretto M., Residori G. 2003, Progetto "I materiali vetrosi nella protostoria dell'Italia del Nord". Archeologia, archeometria , etnoarcheologia e approccio sperimentale, in Bellintani P., Moser L. (a cura di), Archeologie sperimentali. Metodologie ed esperienze fra verifica, riproduzione, comunicazione e simulazione. Atti del convegno (Comano Terme - Fiavè 2011), Trento, pp. 311-335.

Bietti Sestieri A. M., De Santis A. 2004, Analisi delle decorazioni dei contenitori delle ceneri dalle sepolture a cremazione dell'età del Bronzo Finale nell'area centrale tirrenica, in Negroni CATACChio N. (a cura di), Preistoria e Protostoria in Etruria. Miti Simboli Decorazioni Ricerche e scavi, Atti del sesto incontro di studi (Pitigliano - Valentano 2002), I, Milano, pp. 165-192.

Bordes F. 1968, The Old Stone Age, New York, Toronto.

BradFord J. 1992, Costruzioni di graticciate, legno e zolle erbose, in Singer C., Holmyard E. J., Hall A. R., Williams T. I. (a cura di). Storia della Tecnologia 1. La preistoria e gli antichi imperi (ed. orig. A History of Technology 1, Oxford 1954), Torino, pp. 303-331.

Briggs M. S. 1993, Costruzione di edifici, in Singer C., Holmyard E. J., Hall A. R., Williams T. I. (a cura di). Storia della Tecnologia 2. Le civiltà mediterranee e il Medioevo (ed. orig. A History of Technology 2, Oxford 1956), Torino, pp. 404455.

Broholm H. C., Larsen W. P., Skjerne G. 1949, The Lures of the Bronze Age, Copenaghen.

Chevillot C. 1994, Le Parc Archeologique de Beynac, un util pédagogique et de communication, in Les sites de reconstitutions archéologiques, Actes du Colloque (Aubechies 1993), Aubechies, pp. 36-40.

Coles J. 1973, Archaeology by Experiment, London.

Comis L. 2004, Archeologia sperimentale come strumento di ricerca. L'asportazione deivasi dal tornio e lo 'stacco a cordicella'. Il caso delle ciotole rinascimentali di Favaletto (Parma), in Padusa XL, pp. 229-243.

Daniel G. 1968, L'idea della preistoria, (ed. orig. The Idea of Prehistory, London 1962), Firenze.

De Santis A., Merlo R., De Grossi Mazzorin J. 1998, Fidene. Una casa dell'età del Ferro, Milano.

Dobres M. A. 2000, Technology and Social Agency, Oxford.

Ellmers D. 2005, Schiffsarchäologische Experimente in Deutschland, in Experimen-

Conimbriga, 51 (2012) 5-33 
telle Archäologie in Europa 1. Von der Altsteinzeit über "Ötzi” bis zum Mittelalter, Ausgewählte Beiträge zur Experimentellen Archäologie in Europa von 1990-2003, Bad Langensalza/Thüringen, pp. 49-60.

EXARC 2002, Archäologische Freilichtsmuseen in Europa - Archaeological Open Air Museums in Europe, Unteruhldingen.

Frère-SAUTOT M.-C. 2011, Vingt-cinq ans de paléo métallurgie du bronze et du cuivre, in Giardino C. (a cura di), Archeometallurgia: dalla conoscenza alla fruizione. Atti del Workshop (Cavallino-LE 2006), Beni Archeologici - Conoscenza e Tecnologie Quaderno 8, Edipuglia, Bari 2011, pp. 347-353.

Galilei G. 1638, Discorsi e dimostrazioni matematiche intorno a due nuove scienze attenenti alla mecanica \& $i$ movimenti locali, Leida.

Grueber H. A. 1910, Coins of the Roman Republic in the British Museum, III, Oxford. Guidi A. 1988, Storia della Paletnologia, Roma-Bari.

Hahne H. 1919, Das Steinzeithaus zu Rössen, Magdeburg.

Heyerdahl T. 1971, The Ra Expeditions, London.

IngSTAD A. S., 1977, The discovery of a Norse settlement in America, Oslo.

INGSTAD H. 1985, Historical background and the evidence of the site at L'Anse aux Meadows, Oslo.

Leroi-Gourhan A. 1964, La Geste et la Parole 1: Technique et Langage, Paris.

LEUZINGER U. 2002, Experimental and applied archaeology in lake-dwelling research, in Menotti F. (a cura di), Living on the lake in Prehistoric Europe. 150 years of lake dwelling research, London - New York, pp. 237-250.

LubBock J. 1865, Pre-Historic Times, As Illustrated by Ancient Remains, and the Manners and Customs of Modern Savages (I ed.), London.

Lubbock J. 1900, Pre-Historic Times, As Illustrated by Ancient Remains, and the Manners and Customs of Modern Savages (VI ed.), London, Edinburgh, Oxford.

MATHIEU J.R. 2002, Experimental archaeology, replicating past objects, behaviors and processes, BAR International Series 1035, Oxford.

NiELSEN S. 1966, Eksperiment, Skalk 3, pp. 13-23.

NiLSSON S. 1868, The primitive inhabitants of Scandinavia: An essay on comparative ethnography, and a contribution to the history of the development of mankind: containing a description of the implements, dwellings, tombs, and mode of living of the savages in the north of Europe during the stone age, London.

Nydal R. 1989, A critical review of radiocarbon dating of a Norse settlement at L'Anse aux Meadows, Newfoundland, Canada, Radiocarbon 31, pp. 976-985.

Piggott S. 1959, The Carnyx in early Iron Age Britain, in Antiquaries Journal 39, pp. 19-32.

Schmidt M. 1994, Are dull reconstructions more scientific ?, in Les sites de reconstitutions archéologiques, Actes du Colloque (Aubechies 1993), Aubechies, pp. 16-21.

SнӧвеL G. 2004 a, On the responsabilities of accurately interpreting prehistoric life in full scale, in EuroREA - (Re)construction and Experiment in Archaeology 1, pp. 150-160.

Conimbriga, 51 (2012) 5-33 
SнӧвеL G. 2004 b, Lake-dwelling museums. Academic research and public information, in Menotтi F. (a cura di), Living on the lake in Prehistoric Europe. 150 years of lake dwelling research, London - New York, pp. 221-236.

StRöBel R.1939, Die vorgeschichtlichen Freilichtmuseen des Reichsbundes für deutsche Vorgeschichte, in Volk und Vorzeit 2, pp. 42-47.

Sutherland C. H. V. 1974, Roman Coins, Fribourg.

Thomson R. H. G. 1993, L'artigianato medioevale, in Singer C., Holmyard E. J., Hall A. R., Williams T. I. (a cura di). Storia della Tecnologia 2. Le civiltà mediterranee e il Medioevo (ed. orig. A History of Technology 2), Torino, pp. 389-403.

Vecchio G., Albore Livadie C., Castaldo N. 2002, Nola Quattromila anni fa. Il villaggio dell'Età del Bronzo Antico distrutto dal Vesuvio, Nola. 


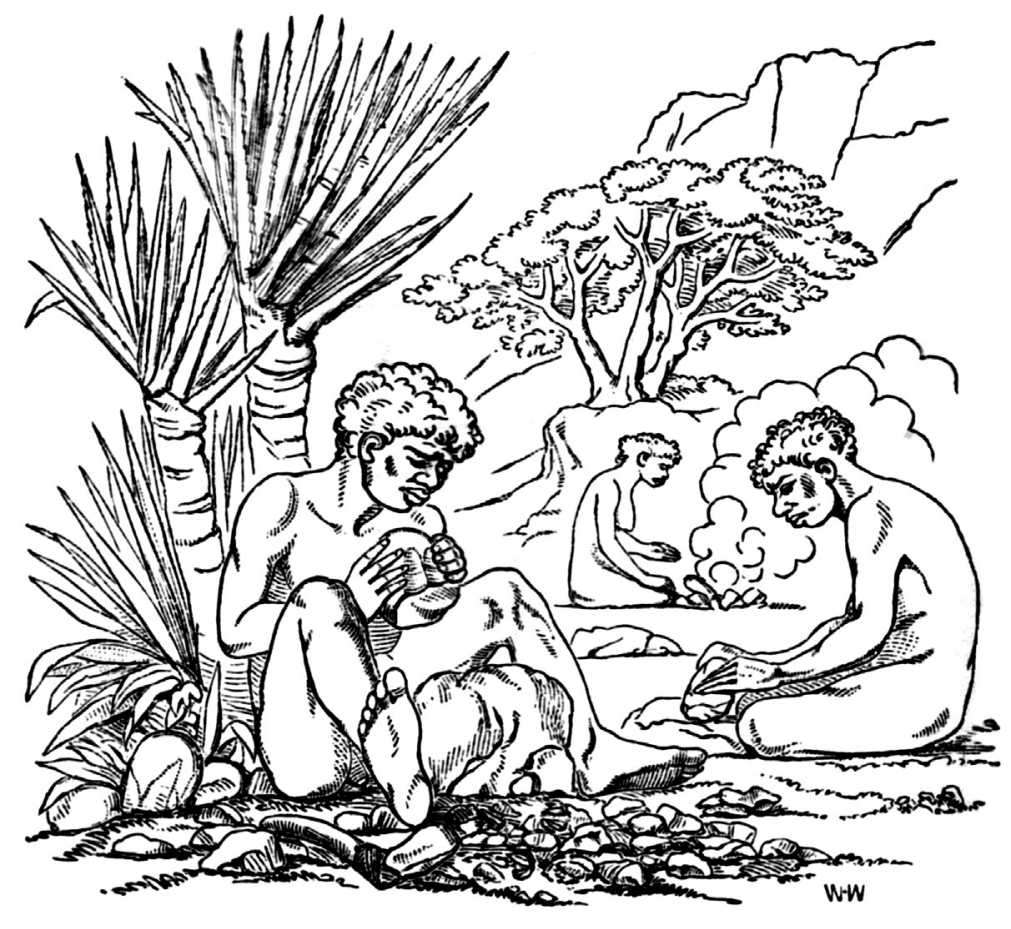

Australians making Flakes.

FIG. 1 - Incisione vittoriana raffigurante la produzione di strumenti litici da parte di aborigeni australiani (da Lubbock 1900). 


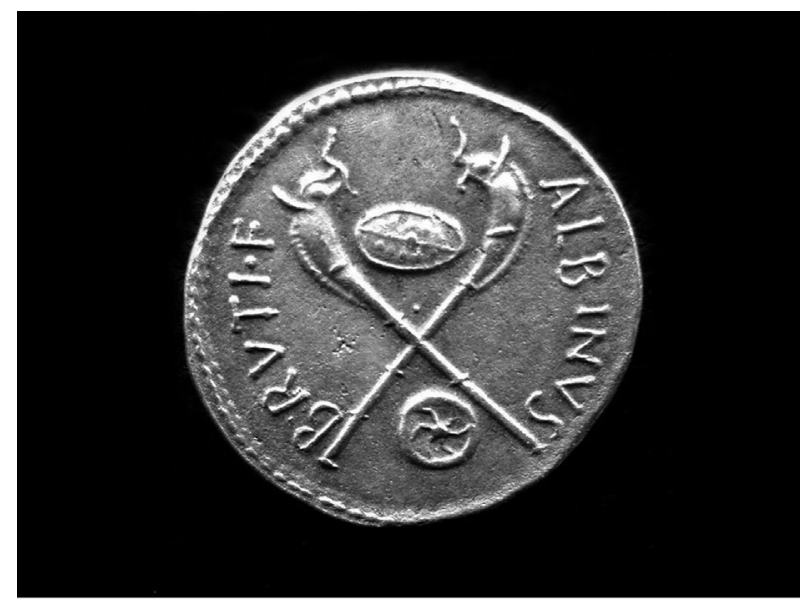

FIg. 2 - Denario coniato da D. Iunius Brutus Albinus riproducente una coppia di carnyx.

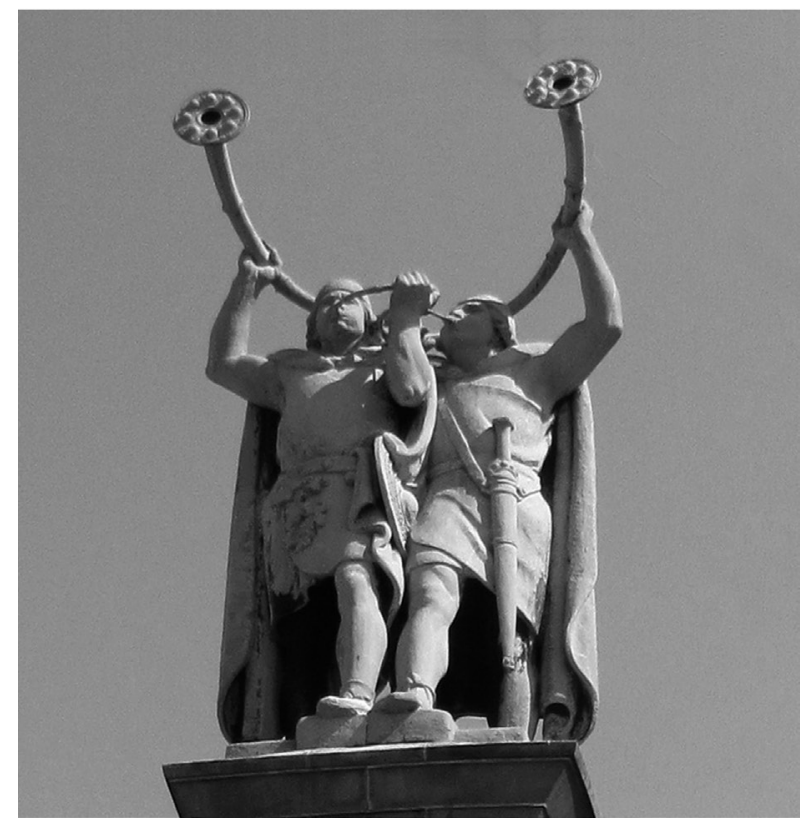

FIG. 3 - Statua con coppia di suonatori di lurer: la "Lurblæserne", eretta a Copenaghen nel 1914. 


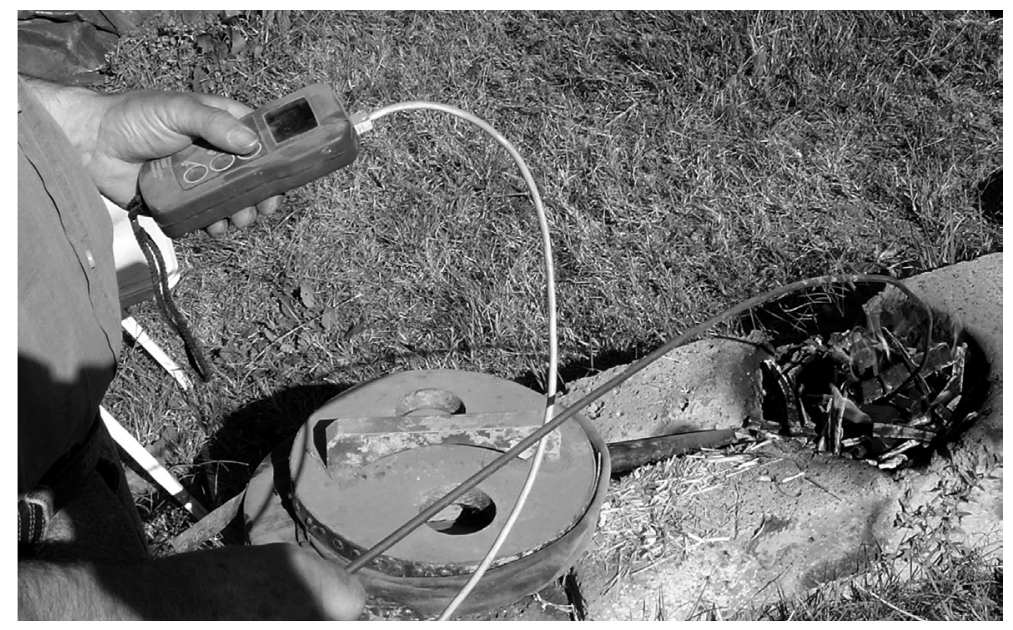

FIG. 4 - Misurazione con termocoppia delle temperature raggiunte in un esperimento per l'estrazione dell'antimonio (Blera - Antiquitates 2011) (Foto C. Giardino).

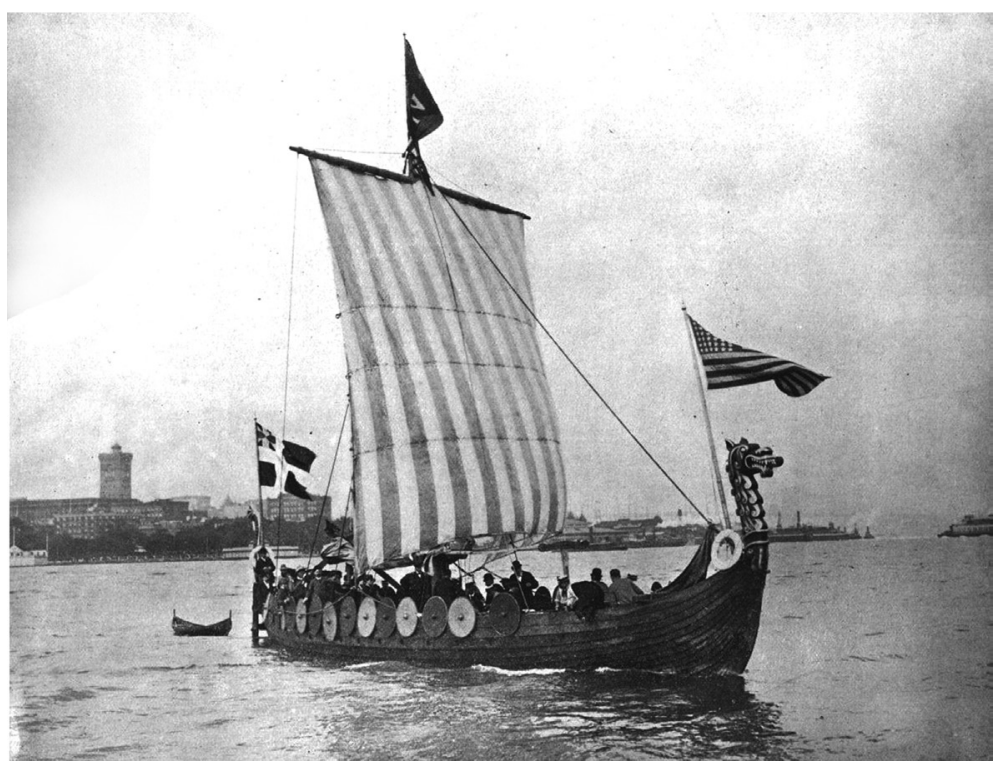

FIG. 5 - La nave Viking in navigazione (1883). 


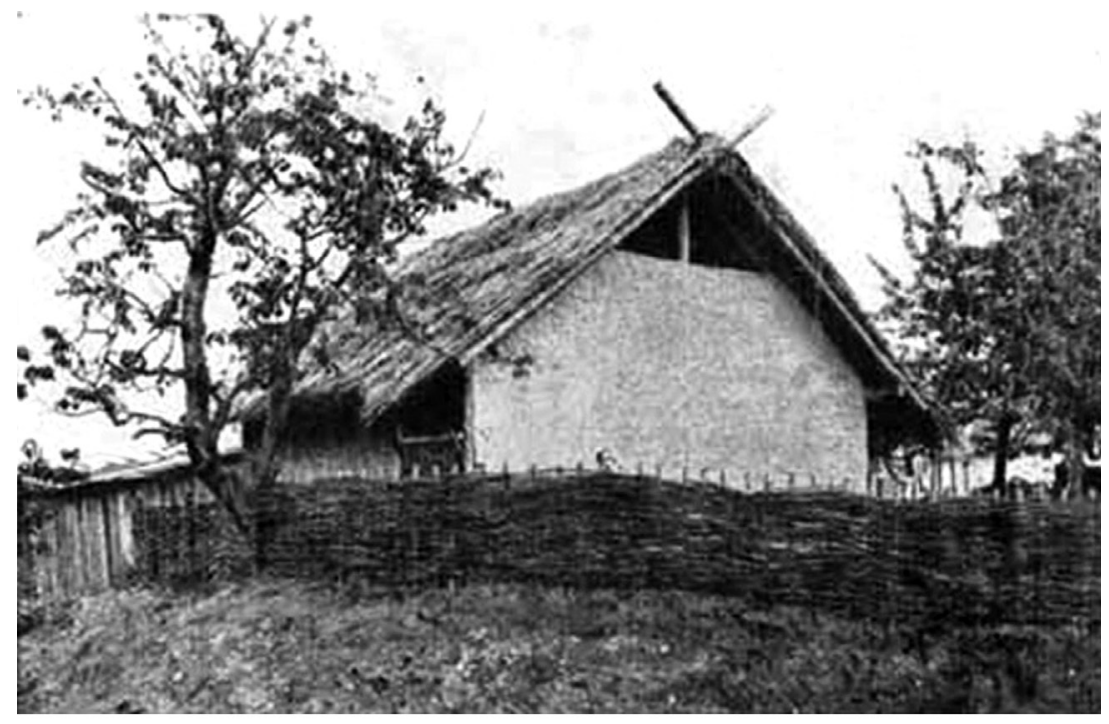

FIG. 6 - La casa neolitica di Rössen (1918) (da una cartolina postale dell'epoca).

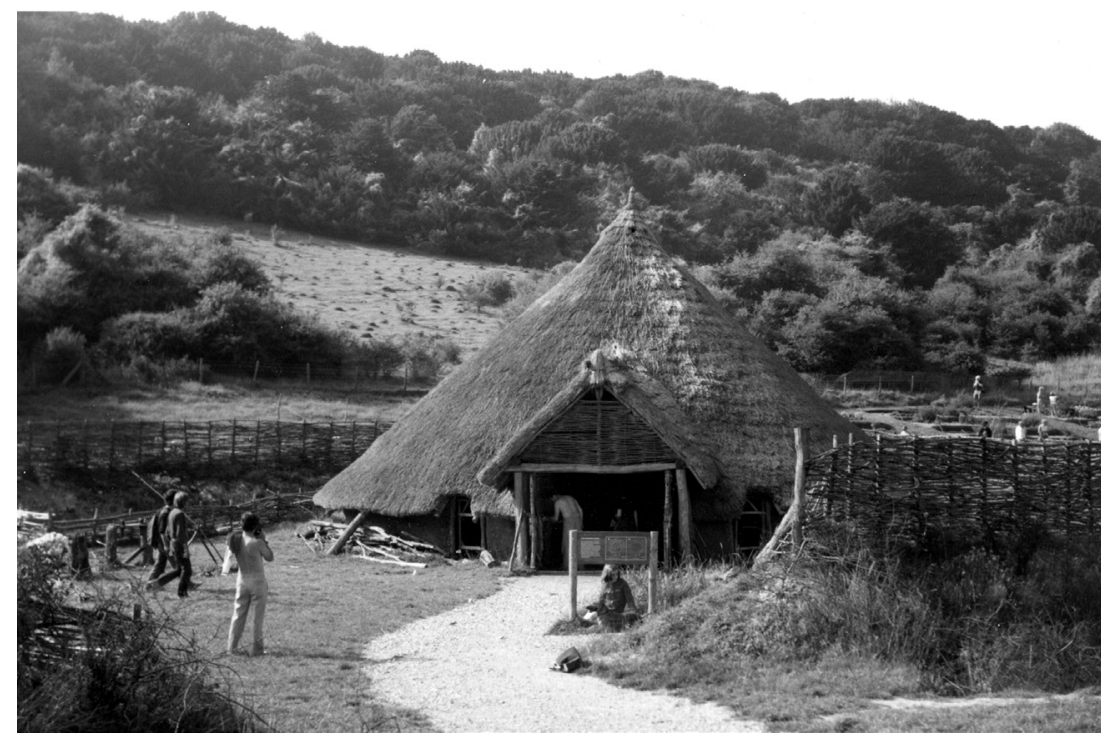

FIg. 7 - Capanna protostorica della Butser Ancient Farm (1979) (Foto C. Giardino). 


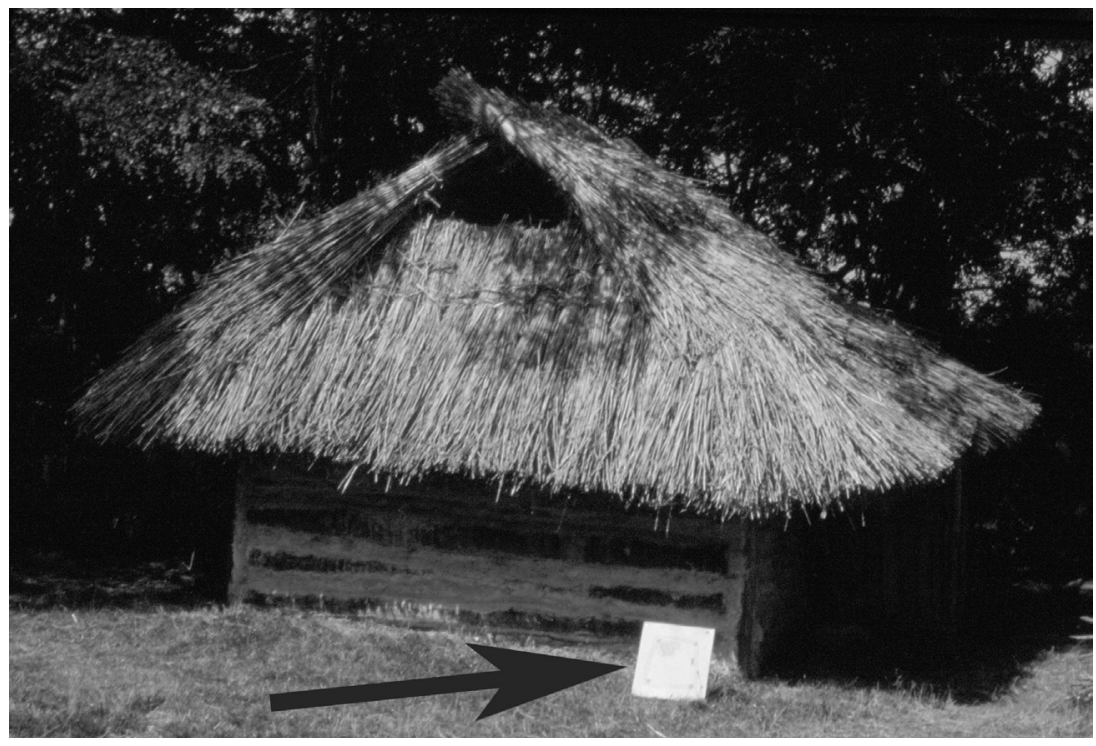

FIG. 8 - Casa dell'età del bronzo ricostruita a Brežno u Loun (1991): la freccia indica il cartello alla base della struttura che illustra la sua pianta con le evidenze archeologiche in base alle quali è avvenuta la ricostruzione (Foto C. Giardino).

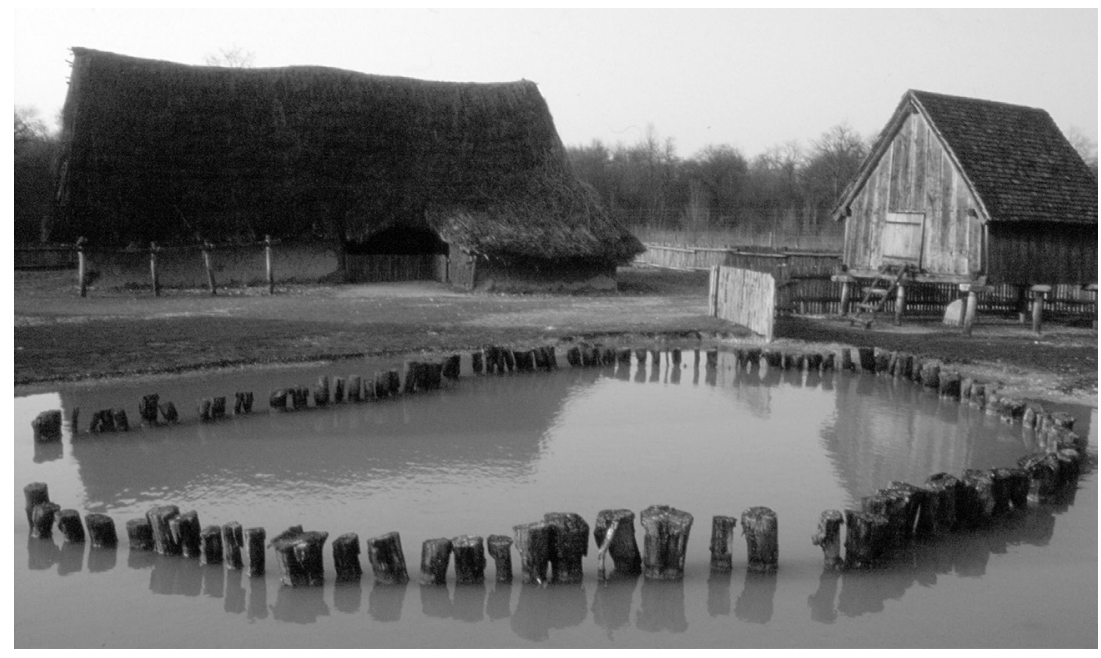

FIG. 9 - Ricostruzione di abitazioni galliche del I secolo a.C. sulla base degli scavi condotti nella valle dell'Aisne nell'Archéodrome di Beaune (1996) (Foto C. Giardino). 


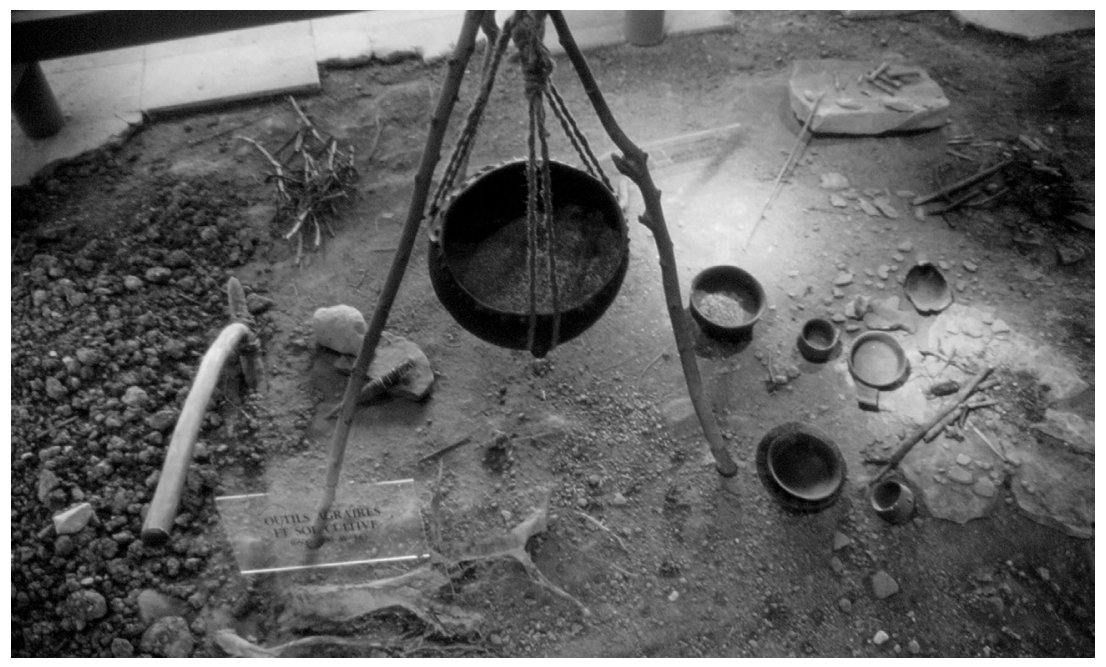

FIG. 10 - Interno di una struttura neolitica riprodotto nel museo dell'Archéodrome di Beaune (1996) (Foto C. Giardino).

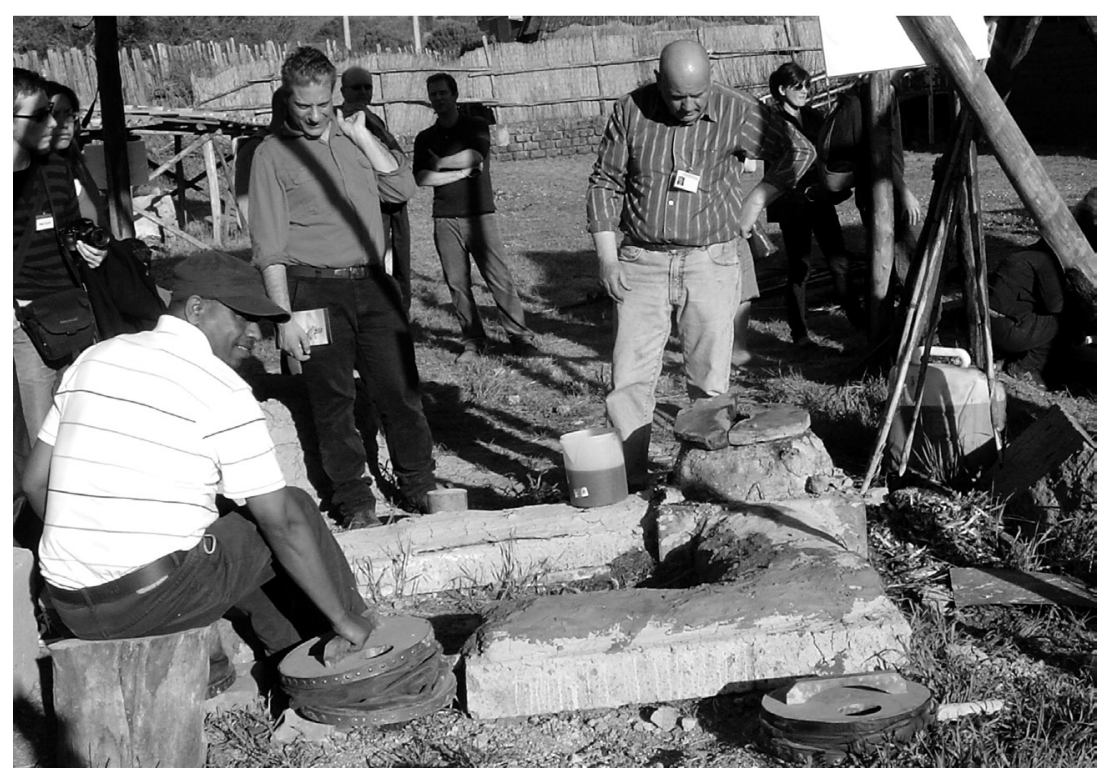

FIG. 11 - Sperimentazioni fusorie al Convegno di Archeometallurgia Sperimentale tenuto al Centro Antiquitates di Blera nel 2011 (Foto C. Giardino). 


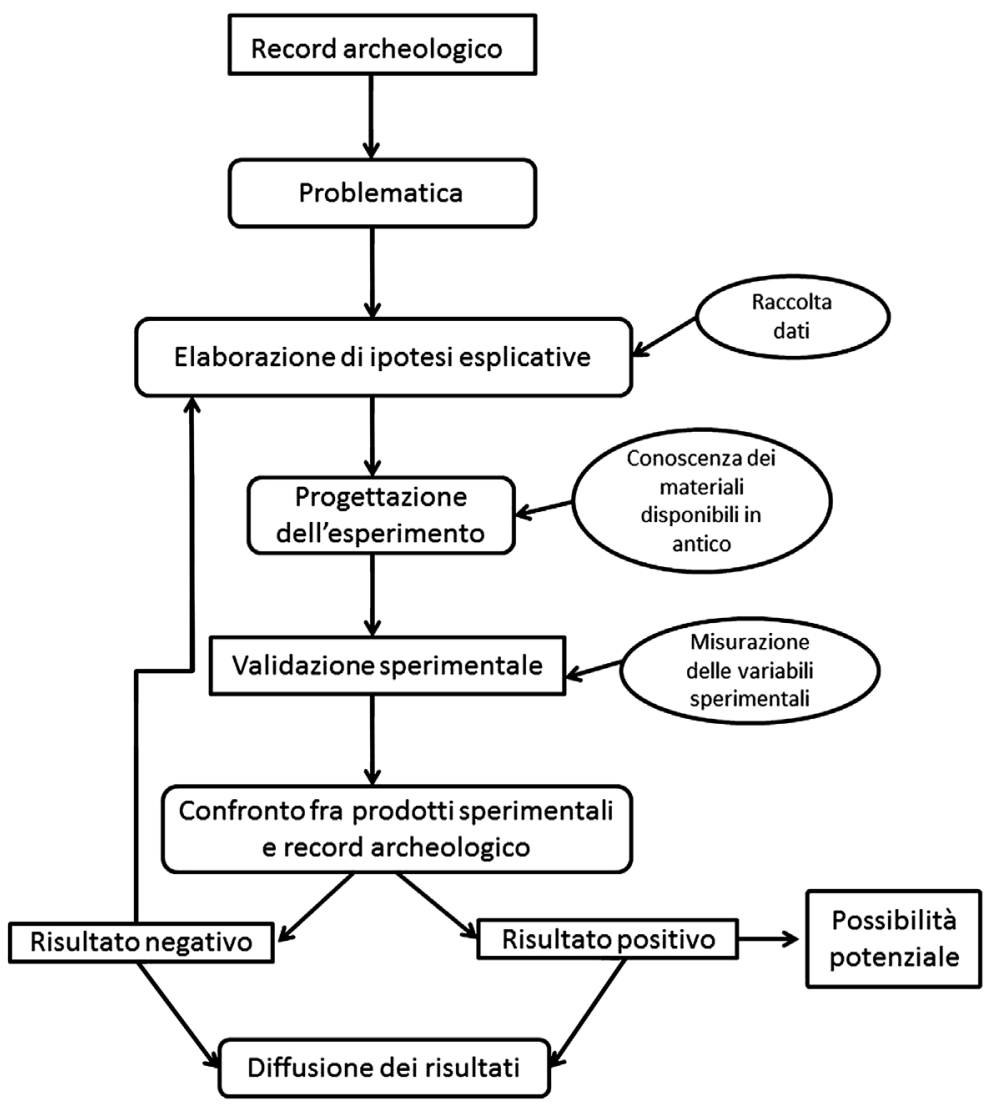

ТАВ. 1 - L'esperimento archeologico. 